\title{
Relationship between the night shift work and thyroid disorders: A systematic review and meta-analysis
}

\author{
Luca Coppeta ${ }^{1}$, Luca Di Giampaolo ${ }^{2}$, Stefano Rizza ${ }^{3}$, Ottavia Balbi ${ }^{1}$, Savino Baldi ${ }^{1}$, \\ Antonio Pietroiusti ${ }^{1}$, Andrea Magrini ${ }^{1}$
}

Italian study group on the metabolic and immunological effects of night shift work; ${ }^{1}$ Department of Occupational Medicine, University of Rome Tor Vergata, Rome, Italy; ${ }^{2}$ Department of Occupational Medicine, University of Chieti G. D’Annunzio, Chieti, Italy; ${ }^{3}$ Department of Systems Medicine, University of Rome Tor Vergata, Rome, Italy E-mail: savino.baldi@hotmail.it

Objectives. The night shift workers were reported to have health consequences, ranging from mild, as cluster headache, to severe, as heart attacks and hormonal irregularities. This study is aimed to perform a systematic review and meta-analyze of the association between the night shift work and the thyroid disorders.

Methods. We comprehensively searched eight databases, including PubMed and Google Scholar for the relevant articles. This systematic review and meta-analysis were conducted according to the Preferred Reporting Items for Systematic Reviews and Meta-analysis (PRISMA) statement.

Results. We finally included six papers involving 4074 participants. Four papers were eligible for meta-analysis involving 1864 night shift workers and 2017 day shift workers. We against found that thyroid stimulating hormone (TSH) is significantly higher in the night shift group compared to the day shift group.

Conclusions. The higher TSH among the night shift workers is attributed to disruption of the circadian rhythm and sleep/wake cycle, with subsequent eating disorders. We proposed that more attention should be paid to the working pattern and the related health consequences.

Key words: night shift, rotating shift, TSH, thyroid disease, systematic review

The night shift work refers to the employment practice occupying a definite time period between 10:00 p.m. and 6:00 a.m. (Saksvik Lehouillier et al. 2015). The shift work has become common, responding to variations in economic pressure and greater consumer demand for 24-hour services. According to the 2010 U.S. Occupational Health Survey, regular night shift workers account for $15 \%$ of the total labor force in the United States (U.S.), whilst $29 \%$ of the total workers involve in rotating shift (Alterman et al. 2013; Liira et al. 2015). As for the working-age population, a higher prevalence of the night shift working has been found in workers aged 18-29. In addition, the level of the education is negatively linked with the night work rates, i.e. the higher level of educa- tion fits with the lower proportion of night workers. This support that service-related occupations shows the highest prevalence of $63 \%$ (Alterman et al. 2013). Indeed, it is increasingly considered that the night shift work leads to negative health consequences. Any anomalies in the physiological activities that include blood pressure, heart rate, body temperature, hormonal secretions, and oxygen consumption, inevitably lead to disorders ranging from the mild to the severe. Thus, the night shift workers are at a higher risk of acquiring serious cardiovascular, digestive, and metabolic disorders, as well as type 2 diabetes, peptic ulcer, hypertension and heart attack (Gan et al. 2015; Knutsson 2003; Vyas et al. 2012). Furthermore, the night workers more likely have sleep and psycho-

Corresponding author: Savino Baldi, Department of Occupational Medicine, University of Rome Tor Vergata, Rome, Italy; e-mail: savino.baldi@hotmail.it. 
social disorders (Akerstedt et al. 2010). In addition, they consume a greater number of cigarettes, food, coffee, and alcohol to overcome stress. In comparable cases, the person is at risk of obesity, which in turn is detrimental to his health (Ker et al. 2010). The night shift work also may predispose to some family, social, and intellectual disorders (Slanger et al. 2016).

Importantly, night shift workers are at increased risk of developing several types of cancers, principally the hormone dependent types, including breast and ovarian cancers. In 2008, the International Center for Cancer Research (ILC) listed night work as a probable carcinogenic agent. This may be ascribed to disruption of the circadian rhythm, with subsequent hormonal changes, particularly melatonin, which is known for its tumor suppressor effects (Megdal et al. 2005).

Literature data have indicated that other hormones may also be affected, such as testosterone, cortisol, prolactin, and thyroid stimulating hormone (TSH) (Ulhoa et al. 2015). This study aims to systematically review and meta-analyze all the relevant published data during the last ten years to examine the definite association between the night shift work and the thyroid disorders.

\section{Methods}

This systematic review and meta-analysis were conducted according to the Preferred Reporting Items for Systematic Reviews and Meta-analysis (PRISMA) statement (Moher et al. 2009). We did not need any ethical approval regarding our study design and institutional policy, since no patients were involved.

Eligibility criteria. We included all the studies discussing the relationship between the night shift work and the thyroid disorders during the last ten years. Although the shift work is under investigation for many decades, we decided to restrict our analysis to a short timeframe since the 3rd generation assay, which has spread worldwide in the last 10 years, now ensures an excellent analytical sensitivity, able to determine very low TSH concentrations (Roggenbuck et al. 2018). No restrictions were considered regarding age, sex, language, and ethnicity of the population. We excluded editorial comments, thesis, reviews, book chapters, news, and only abstract articles. Articles indicating no relevant or inaccessible data were also excluded.

Search strategy and study selection. We comprehensively searched PubMed, Web of Science (ISI), Google Scholar, Popline, Global Health Library
(GHL), and Virtual Health Library (VHL) including Cochrane database, NYAM (New York Academy of Medicine), and SIGLE (System for Information on Grey Literature in Europe) for the relevant articles from January 2008 to October 2018. We used the following search terms ("night shift" OR "rotating shift" OR "night work" OR "evening work" OR "evening shift") AND ("thyroid" OR "thyroid function" OR “TSH” OR "thyroid disease" OR "thyroid stimulating hormone" OR "hypothyroidism" OR "autoimmune thyroid disease" OR "hyperthyroidism" OR "thyrotropin"). The search string was modified in agreement with each database. As for the retrieval of search results, we used EndNote X7.4 software to remove the duplicates. Title and abstract screening were performed by two independent reviewers, then followed by full-text screening to finally include the relevant articles according to our inclusion and exclusion criteria. Then, we manually searched the references of the included articles to add further relevant studies.

Data extraction. Two independent reviewers extracted the relevant data from the finally included studies. A senior third reviewer resolved any discrepancy. The extraction was performed upon a standardized extraction form. It was formulated through pilot extraction of three of the included studies to show the variables of interest. We extracted variables related to the demographic characteristics of the patients included, including the study comparison, occupation, country, age, gender, and the work duration. We also extracted variables showing the association between the night shift work and the thyroid disorders, including thyroid stimulating hormone concentration (TSH), thyroid hormones concentration (T3 and T4). Prevalence of the thyroid diseases, thyroid cancer and autoimmune thyroiditis were also extracted. Graphically presented data were extracted using PlotDigitizer software (http://plotdigitizer. sourceforge.net/).

Statistical analysis. To assess the effects of the night shift work, the mean difference (MD) of TSH with relative $95 \%$ confidence interval was used for final values of thyroid disorders.

We graphically represent pooled results in forest plots, with point estimates of the effect size and 95\% CIs for each study and overall, being the area of squares proportional to each study weight.

Q statistic and I2 were used to evaluate heterogeneity among studies. I2 statistics quantify the percentage of total variation across the studies attributable to heterogeneity. A p-value of Q statistic $<0.10$ was considered significant. 
Jackknife (leave-one-out) sensitivity analysis was performed to evaluate the robustness of the results and the impact of each single study on the summary estimate of effect: we recalculated pooled estimates, using a random-effects model, each time with the removal of a single study from the original group.

We considered a two-sided p-value $<0.05$ as statistically significant. All analyses were performed using ReviewManager (RevMan version 5.3, The Nordic Cochrane Centre, The Cochrane Collaboration, 2008, Copenhagen, Denmark), and Comprehensive Meta-analysis (version 3, Biostat, 14 North Dean Street Englewood, NJ07631, USA) software packages.

\section{Results}

Search results and characteristics of the included studies. From the initial search of the different databases, we identified 69 studies, 25 of which were duplicates, hence excluded by EndNote. The remaining 44 reports were categorized for title, abstract, and full-text screening. As far as it concerns screening, 6 reports were included for extraction, whereas 38 were excluded for several reasons. The manual search did not show any further papers.

Four papers out of the six were eligible for the meta-analysis (Figure 1). The four papers of the meta-analysis compared the night shift workers (1864 participants) with the day shift workers (2017 participants). The studies included for the metaanalysis show different results as for the duration of the shift working. Shaker et al. (2018) have compared the night and the day shift workers, with a shift working history averaging around 18.9 years for both the groups of subjects, whereas in the study of Moon et al. (2016), the average duration of the shift working was five years. Harbeck et al. (2015) have compared a 24 -hour on-call shift with 7-hour day shift participants, but the study did not indicate the past shift working history for both groups. Korompeli et al. (2009) have compared the night shift workers for 9 years with the day shift workers for 17.5 years. Burdelak et al. (2012) and Chang et al. (2014) were not eligible for analysis. The two papers have assessed thyroid disorders through different variables, which are inconsistent with the other papers. Burdelak et al. (2012) have assessed the prevalence of thyroid diseases, while Korompeli et al. (2009) have assessed $\mathrm{T} 3$ and T4 concentrations.

Outcomes. Thyroid stimulating hormone (TSH) is the variable eligible for the meta-analysis, because it is presented in four papers of the six, i.e. Shaker et al. (2018); Moon et al. (2016); Harbeck et al. (2015);

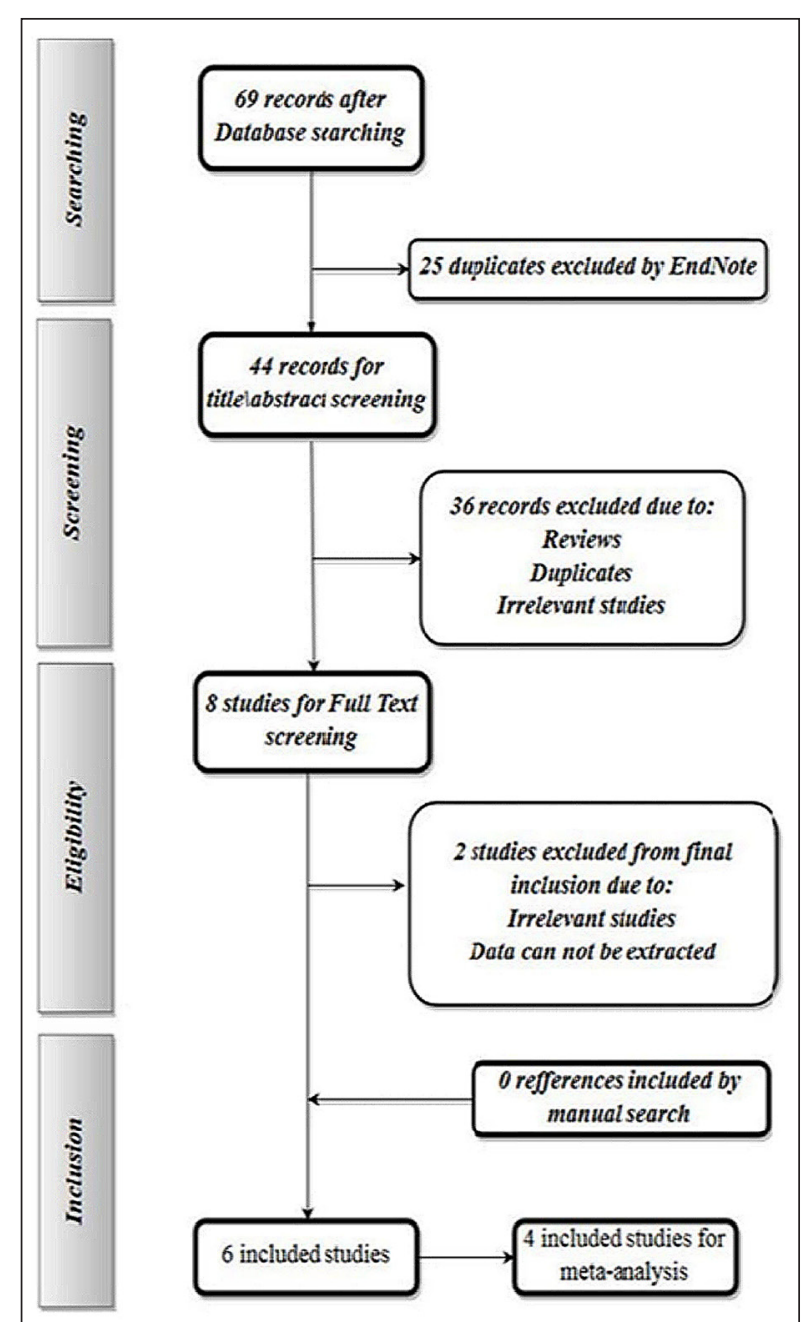

Figure 1. Flow chart illustrating the sequence of the search strategy.

Korompeli et al. (2009). Other variables were presented only in one study and were not consistent with others. Burdelak et al. (2012) have assessed the prevalence of thyroid diseases among 725 participants.

Thyroid diseases account for $21.2 \%$ of the total included population. Fifty-two percent (52\%) of the detected thyroid patients were night shift workers, whilst $48 \%$ of them were day shift workers. The study indicated an increased relative risk of thyroid diseases among the night shift workers but the result is not statistically significant. In addition, Korompeli et al. (2009) have detected the thyroid hormone levels (T3 and T4) among the night and the day shift workers. There was a statistically significant increase of the thyroxine in the rotating shift group but not in the day shift group. As for TSH, it did not show a significant difference between the two groups (MD 
$0.875,95 \% \mathrm{CI}-0.458$ to $2.208, \mathrm{p}=0.198$ ) (Figure 2). However, TSH showed a significant increase in the night shift group against the morning shift group after the removal of results reported by Moon et al. (2016) (MD 1.261, 95\% CI -0.083 to $2.439, \mathrm{p}=0.036$ ) (Figure 3).

\section{Discussion}

This study is the first systematic review and metaanalysis paper discussing the association between the night shift work and the thyroid disorders. It has been described that serum TSH assay is the most sensitive and reliable method to detect thyroid dysfunction and subclinical hypothyroidism (Fatourechi 2007), consequently, the main concern of our study was to detect and compare serum TSH concentrations in the night shift and the day shift groups. Other studies have noticed the prevalence of thyroid diseases among the night shift and the day shift workers. Accordingly, thyroid disorders showed high prevalence rates among the night shift workers, rather than in those taking the day shifts only (Moon et al. 2016). In addition, the night shift work is commonly associated with the sleep related disorders and insomnia. Significantly, an epidemiological study has shown that postmenopausal women, suffering from insomnia, are significantly at a higher risk of developing the thyroid cancer. The risk rises with higher insomnia grades (Luo et al. 2013). Moreover, another study has shown a significant increase of the autoimmune hypo- and hyperthyroidism among the night shift workers. Indeed, it is increasingly appreciated that the night work associated stress induces longterm variation of the immune system (Boscolo et al. 2008). Furthermore, many studies have explored the association between the night shift work and TSH levels. We present a systematic review and meta-analysis of those studies that provide highly evident and reliable results over the separate studies. Our study showed a significant increase of TSH concentrations among the night shift against the day shift workers, after the removal of Moon et al. (2016). This discrepancy may be adjusted by the presence of significant heterogeneity regarding the type and amount of the night shifts. Supporting the fact that the accuracy of the data are questionable, since they have been retrospectively obtained from employees as a medical check-up data. In addition, it has been reported that the night shift work positively correlates with the risk of developing subclinical hypothyroidism. Moreover, the duration of night working, the higher the risk (Sviridonova et al. 2013). Indeed, the most sensitive and reliable screening test for detection of subclinical hypothyroidism is the serum TSH assay. It is estimated that $4-15 \%$ of the general population have subclinical hypothyroidism (Sviridonova et al. 2013). The prevalence rate increases to $25-50 \%$ in the case of presence of anti-thyroid peroxidase autoantibodies (anti-TPO). Positive anti-TPO or/and TSH levels of $>3.0-4.5 \mathrm{mIU} / \mathrm{L}$ increase the risk of progression of subclinical hypothyroidism to overt hypothyroidism (Hennessey and Espaillat 2015). Interestingly, it has been shown that most of the study participants are females, suggesting that females are more sensitive to the night shift working than males. This may be attributed to the variability and sensitivity of the female hormones, including hormone of the menstrual cycle (Lawson et al. 2011). With respect to the management of the subclinical hypothyroidism, it is recommended to start drug treatment if TSH levels $>10 \mathrm{mIU} / \mathrm{L}$ (Hennessey and Espaillat 2015).

It has been suggested that the reported higher TSH in the night shift workers is attributed to disruption of the circadian rhythm (James et al. 2017). Under physiological conditions, TSH shows circadian rhythmicity, with its peak at mid-night (2:00-4:00 a.m.) and trough at (4:00-8:00 p.m.) (Weibel et al. 1995). Significantly, the night shift workers show disturbed sleep/wake cycle, which becomes inconsistent with the biological timing system. The entire body loses its hormonal homeostatic properties and thyroid dysfunction occurs. Supporting that sleep deprivation stimulates the disruption of TSH circadian rhythm (Kessler et al. 2010). Moreover, the night shift workers are more susceptible to irregular eating habits and nocturnal eating. In fact, nocturnal eating is associated with increased levels of hormones, including TSH and insulin (Holmback et al. 2003). Furthermore, stress associated with the night work is suggested to have long-term modulating impact on the immune system (Boscolo et al. 2008). Therefore, even if the mechanisms generating ultradian TSH rhythms are still unclear (Ikegami et al. 2019), it is conceivable that the increased TSH levels in night shift workers can reflect desynchronized rhythm. Furthermore, stress associated with the night work is suggested to have long-term modulating impact on the immune system and on thyroid oncogenesis (Rizza et al. 2020). Consequently, anti-TPO is more likely to be present, which carries a higher risk of developing subclinical and overt hypothyroidism (Magrini et al. 2006).

Strengths. This is the first systematic review and meta-analysis discussing the relationship between the night shift work and the thyroid disorders. Our 


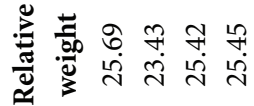

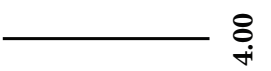

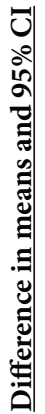
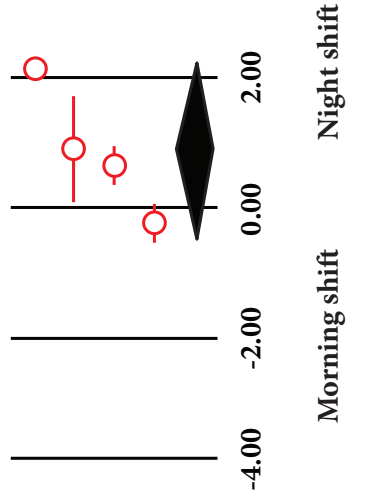

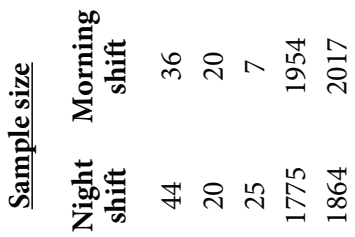

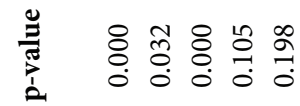

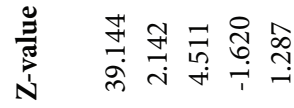

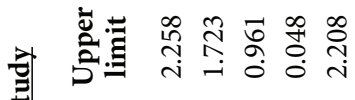

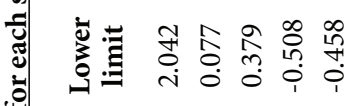

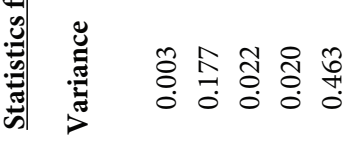

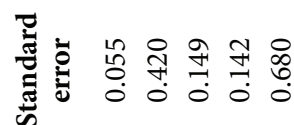

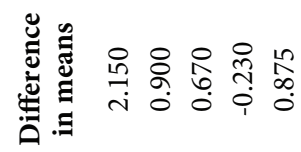

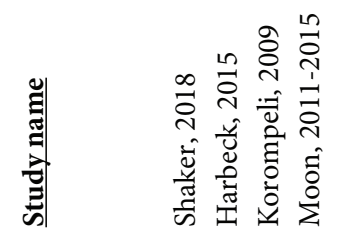

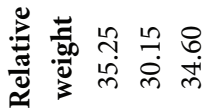

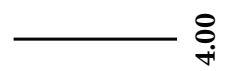

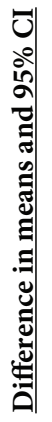

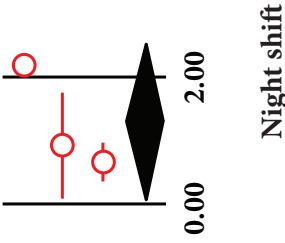

咅

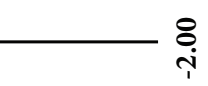

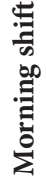

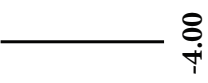

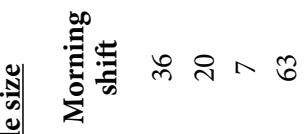

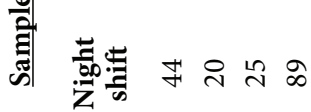

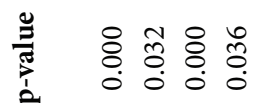

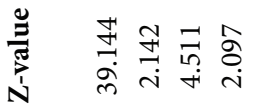

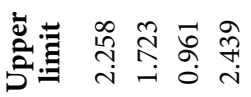

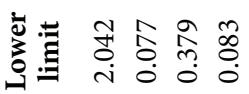

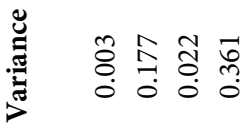

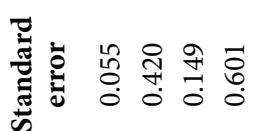

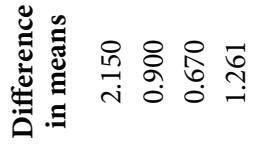

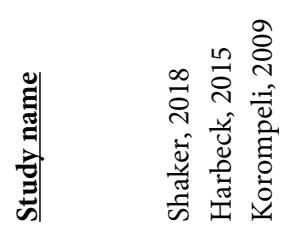


study shows more evident and reliable results than the separate studies.

Limitations. Most of the included studies included only females, so the evaluation of male workers was not available. Blood samples were taken in different moments and the TSH circadian rhythm was not considered. In addition, the sample size was relatively small. One of our limitations was the low number of the included studies eligible for analysis.

\section{Conclusions}

We found that thyroid disorders were more prevalent among the night shift workers. The night work was more associated with increased TSH levels. The night shift workers were at a higher risk of developing subclinical hypothyroidism. More attention should be paid to the working pattern and its related health consequences. Further quality research is needed to thoroughly discuss the association between the night shift work and its duration with the thyroid disorders.

\section{Acknowledgements}

This research did not receive any specific grant from any funding agency from the public, commercial or not-for-profit sectors. The authors confirm that the data supporting the findings of this study are available within the article.

\section{References}

Akerstedt T, Nordin M, Alfredsson L, Westerholm P, Kecklund G. Sleep and sleepiness: Impact of entering or leaving shiftwork - A prospective study. Chronobiol Int 27, 987-996, 2010.

Alterman T, Luckhaupt SE, Dahlhamer JM, Ward BW, Calvert GM. Prevalence rates of work organization characteristics among workers in the U.S.: Data from the 2010 National Health Interview Survey. Am J Ind Med 56, 647-659, 2013.

Boscolo P, Youinou P, Theoharides TC, Cerulli G, Conti P. Environmental and occupational stress and autoimmunity. Autoimmun Rev 7, 340-343, 2008.

Burdelak W, Bukowska A, Krysicka J, Peplonska B. Night work and health status of nurses and midwives. crosssectional study. Med Pr 63, 517-529, 2012.

Chang YS, Chen HL, Wu YH, Hsu CY, Liu CK, Hsu C. Rotating night shifts too quickly may cause anxiety and decreased attentional performance, and impact prolactin levels during the subsequent day: a case control study. BMC Psychiatry 14, 218, 2014.

Fatourechi V. Upper limit of normal serum thyroid-stimulating hormone: a moving and now an aging target? J Clin Endocrinol Metab 92, 4560-4562, 2007.

Gan Y, Yang C, Tong X, Sun H, Cong Y, Yin X, Li L, Cao S, Dong X, Gong Y, Shi O, Deng J, Bi H, Lu Z. Shift work and diabetes mellitus: A meta-analysis of observational studies. Occup Environ Med 72, 72-78, 2015.

Harbeck B, Suefke S, Haas CS, Lehnert H, Kropp P, Moenig H. No stress after 24-hour on-call shifts? J Occup Health 57, 438-447, 2015.

Hennessey JV, Espaillat R. Diagnosis and management of subclinical hypothyroidism in elderly adults: A review of the literature. J Am Geriatr Soc 63, 1663-1673, 2015.

Holmback U, Forslund A, Lowden A, Forslund J, Akerstedt T, Lennernas M, Hambraeus L, Stridsberg M. Endocrine responses to nocturnal eating - possible implications for night work. Eur J Nutr 42, 75-83, 2003.

Ikegami K, Refetoff S, Van Cauter E, Yoshimura T. Interconnection between circadian clocks and thyroid function. Nat Rev Endocrinol 15, 590-600, 2019.

James SM, Honn KA, Gaddameedhi S, Van Dongen HPA. Shift work: disrupted circadian rhythms and sleep-implications for health and well-being. Curr Sleep Med Reports 3, 104-112, 2017.

Ker K, Edwards PJ, Felix LM, Blackhall K, Roberts I. Caffeine for the prevention of injuries and errors in shift workers. Cochrane Database Syst Rev 2010, CD008508, 2010.

Kessler L, Nedeltcheva A, Imperial J, Penev PD. Changes in serum TSH and free T4 during human sleep restriction. Sleep 33, 1115-1118, 2010.

Knutsson A. Health disorders of shift workers. Occup Med (Lond) 53,103-108, 2003.

Korompeli A, Sourtzi P, Tzavara C, Velonakis E. Rotating shift-related changes in hormone levels in intensive care unit nurses. J Adv Nurs 65, 1274-1282, 2009.

Lawson CC, Whelan EA, Lividoti Hibert EN, Spiegelman D, Schernhammer ES, Rich-Edwards JW. Rotating shift work and menstrual cycle characteristics. Epidemiology 22, 305-312, 2011. 
Liira J, Verbeek JH, Costa G, Driscoll TR, Sallinen M, Isotalo LK, Ruotsalainen JH. Pharmacological interventions for sleepiness and sleep disturbances caused by shift work. Sao Paulo Med J 133, 67, 2015.

Luo J, Sands M, Wactawski-Wende J, Song Y, Margolis KL. Sleep disturbance and incidence of thyroid cancer in postmenopausal women the Women's Health Initiative. Am J Epidemiol 177, 42-49, 2013.

Magrini A, Pietroiusti A, Coppeta L, Babbucci A, Barnaba E, Papadia C, Iannaccone U, Boscolo P, Bergamaschi E, Bergamaschi A. Shift work and autoimmune thyroid disorders. Int J Immunopathol Pharmacol 19, 31-36, 2006.

Megdal SP, Kroenke CH, Laden F, Pukkala E, Schernhammer ES. Night work and breast cancer risk: A systematic review and meta-analysis. Eur J Cancer 41, 2023-2032, 2005.

Moher D, Liberati A, Tetzlaff J, Altman DG; PRISMA Group. Preferred reporting items for systematic reviews and meta-analyses: the PRISMA statement. PLoS Med 6, e1000097, 2009.

Moon SH, Lee BJ, Kim SJ, Kim HC. Relationship between thyroid stimulating hormone and night shift work. Ann Occup Environ Med 28, 53, 2016.

Rizza S, Neri A, Capanna A, Grecuccio C, Pietroiusti A, Magrini A, Federici M, Coppeta L. Night shift working is associated with an increased risk of thyroid nodules. J Occup Environ Med 62, 1-3, 2020.

Roggenbuck JJ, Veiczi M, Conrad K, Schierack P, Wunderlich G, Kotzerke J, Roggenbuck D, Zophel K. A novel thirdgeneration TSH receptor antibody (TRAb) enzyme-linked immunosorbent assay based on a murine monoclonal TSH receptor-binding antibody. Immunol Res 66, 768-776, 2018.

Saksvik Lehouillier I, Pallsen S, Biorvatin B, Mageroy N, Folkard S. Towards a more comprehensive definition of shift work tolerance. Ind Health 53, 69-77, 2015.

Shaker D, Samir A, Zyada F, El-Sharkawy M, Ekladious SM. Impact of shift work on sleep problems, hormonal changes, and features of metabolic syndrome in a sample of Egyptian industrial workers: A cross-sectional study. Middle East Curr Psychiatry 25, 91-97, 2018.

Slanger TE, Gross JV, Pinger A, Morfeld P, Bellinger M, Duhme AL, Reichardt Ortega RA, Costa G, Driscoll TR, Foster RG, Fritschi L, Sallinen M, Liira J, Erren TC. Person-directed, non-pharmacological interventions for sleepiness at work and sleep disturbances caused by shift work. Cochrane Database Syst Rev 2016, CD010641, 2016.

Sviridonova MA, Fadeyev VV, Sych YP, Melnichenko GA. Clinical significance of TSH circadian variability in patients with hypothyroidism. Endocr Res 38, 24-31, 2013.

Ulhoa MA, Marqueze EC, Burgos LG, Moreno CR. Shift work and endocrine disorders. Int J Endocrinol 2015, $826249,2015$.

Vyas MV, Garg AX, Iansavichus AV, Costella J, Donner A, Laugsand LE, Janszky I, Mrkobrada M, Parraga G, Hackam DG. Shift work and vascular events: Systematic review and meta-analysis. BMJ 345, e4800, 2012.

Weibel L, Brandenberger G, Goichot B, Spiegel K, Ehrhart J, Follenius M. The circadian thyrotropin rhythm is delayed in regular night workers. Neurosci Lett 187, 83-86, 1995. 